\title{
Autophagy-related protein LC3 and Beclin-1 in the first trimester of pregnancy
}

\author{
Barbara Chifenti ${ }^{*}$, Maria Teresa Locci ${ }^{1 *}$, Gloria Lazzeri' , Mariangela Guagnozzi ${ }^{1}$, Dino Dinucci ${ }^{2}$, Federica Chiellini², \\ Maria Elena Filice ${ }^{3}$, Maria Giovanna Salerno ${ }^{4}$, Lorella Battini ${ }^{4}$ \\ 'Department of Translational Research on New Technologies in Medicine and Surgery, University of Pisa Medical School, Pisa; ${ }^{2}$ BIOlab, Department of \\ Chemistry and Industrial Chemistry, University of Pisa, Pisa; ${ }^{3}$ Anatomy and Pathology Department, Section 2 Santa Chiara Hospital-University Health \\ Care System, Pisa; ${ }^{4}$ Obstetrics and Gynecology Unit 2, Santa Chiara Hospital-University Health Care System, Pisa, Italy
}

\begin{abstract}
Autophagy is a degradation process that acts in response to environmental stressors. Recently, autophagy has been detected in normal term, preeclamptic and intrauterine growth-restricted placentas. The object of this work was to investigate the presence of autophagy in first trimester voluntary interruption of pregnancy placental villi by the expression of autophagy-related proteins, light chain 3 (LC3), and Beclin-1. In first trimester placental villi laser scanning confocal microscopy (LSCM) analysis revealed LC3 and Beclin-1 immunoreactivity prevalently located in villous cytotrophoblasts. Using LSCM, LC3, and Beclin-1 were localized to the cytoplasm of the trophoblast layer in human full-term placentas. Beclin-1 expression and LC3 activation were confirmed by western blotting. These data emphasize that autophagy activation is different among cytotrophoblasts and syncytiotrophoblasts depending on the gestational age and thus we speculate that autophagy might play a prosurvival role throughout human pregnancy.
\end{abstract}

Keywords: Autophagy; Light chain 3; Beclin-1; Villi; Cytotrophoblasts; Syncytiotrophoblasts

\section{Introduction}

Autophagy is an evolutionarily conserved process in which a portion of cytoplasm is sequestered in a double-membraned autophagosome and subsequently degraded upon fusion with a lysosome [1]. Autophagy occurs to perform homeostatic functions, and recent studies have also demonstrate autophagy involvement in general processes such as development, differentiation, aging and cell death [2]. The hallmark of autophagy is the formation of a double-mem-

Received: Nov 23, 2012 · Revised: Dec 6, 2012 · Accepted: Feb 14, 2013 Corresponding author: Maria Teresa Locci

Department of Translational Research on New Technologies in Medicine and Surgery, University of Pisa Medical School, via Roma 55, 56126 Pisa, Italy Tel: +39-050-2218552 Fax:+39-050-2218557

E-mail:mariateresa.locci@med.unipi.it

*These authors contribute equally to this work.

*This study was supported, in part, by a grant from the Associazione Rinascita Pisana, Pisa, Italy.

This is an Open Access article distributed under the terms of the Creative Commons Attribution Non-Commercial License (http://creativecommons.org/licenses/by-nc/3.0/) which permits unrestricted non-commercial use, distribution, and reproduction in any medium, provided the original work is properly cited. brane cytosolic vesicle, the autophagosome, which sequesters cytoplasm and delivers it to the lysosomes for degradation. The execution of autophagy involves a set of products, known as autophagy-related proteins (ATG). Among these proteins, the yeast ATG-8 mammalian homolog microtubule-associated protein 1 light chain 3 (LC3) is essential for final autophagosome formation [3]. LC3 exists in two forms: a cytosolic form (LC3-I) and a lipid phosphatidylethanolamine-conjugated form (LC3-II) that is inserted into both inner and outer membranes of the growing autophagosome. Therefore, $\mathrm{LC} 3$ can be used as a marker of autophagy by immunofluorescence studies and the monitoring of LC3-I and LC3-II by western blotting.

The mammalian autophagy gene Beclin-1, or yeast homologue Atg6, was cloned through a yeast two-hybrid screen in 1998 by Beth Levine's group [4]. Through its interaction with class III phosphatidylinositol-3-phosphate kinase (PI3kIII/ Vps34), Beclin-1 regulates autophagy. In contrast to $\mathrm{LC} 3$, a marker of final autophagosome formation, Beclin-1 participates in the early stages of autophagy, promoting the nucleation of the autophagic vesicle and recruiting proteins from the cytosol. Recently, Beclin-1 has been shown experimentally 
to be a haploinsufficient tumor suppressor, being monoallelically deleted in some tumors such as those of the breast, ovarian, and prostate [5-7]. Furthermore, Beclin-1 is implicated in numerous biological processes, including adaptation to stress, development, endocytosis, cytokinesis, immunity, tumorigenesis, aging, and cell death [8].

The human placenta is a transient organ that provides nutrition, gas exchange, and immune support for the developing fetus. In the first trimester of human pregnancy, the chorionic villi of the placenta are large and covered by a multinucleated and terminally differentiated epithelium, the syncytiotrophoblast, with a subjacent layer of mononuclear cytotrophoblast stem cells attached to the basement membrane that surrounds the stromal core of the chorionic villi [9]. The syncytiotrophoblast layer has a short lifespan; therefore, cytotrophoblasts continuously proliferate, differentiate and fuse with the covering syncytiotrophoblasts.

To date, autophagy has been studied in normal term placentas in relation to labor [10] and increased autophagy induction has been detected in placentas of preeclamptic [11] and intrauterine growthrestricted pregnancies compared to normal controls [12,13]. Prompted by these findings, we set out to examine the presence of autophagy-related proteins, LC3 and Beclin-1, in human first trimester placental villi by laser scanning confocal microscopy (LSCM) and western blotting studies.

Term placental villi were also analyzed for the same autophagic markers.

\section{Methods}

\section{Collection of placental samples}

Human first trimester placental samples were obtained from voluntary interruptions of pregnancy at 8 to 12 weeks $(n=10)$. Ten placentas from women who had elective caesarean deliveries before the onset of labor were also collected. None of the subjects included in this study reported any medical or pregnancy complications.

Prior to enrolment, the women participating in this study provided their written informed consent. In addition, the local research ethics committee approved this protocol.

The tissues were fixed in 10\% buffered formalin and embedded in paraffin. Sections of $4 \mu \mathrm{m}$ were cut and mounted on polylysine coated slides. For western blotting, tissues were snap frozen and stored at $-80^{\circ} \mathrm{C}$ until protein extraction.

\section{Immunofluorescence labelling}

Paraffin-embedded tissue sections were dewaxed in xylene and rehydrated through a graded series of alcohols. LC3 and Beclin-1 were detected using a rabbit polyclonal anti-LC3 antibody (1:300) (NB1002220, Novus Biologicals, Littleton, CO, USA) and a rabbit polyclonal
anti-Beclin-1 antibody (1:50) (Santa Cruz Biotechnology Inc., Santa $\mathrm{Cruz}, \mathrm{CA}, \mathrm{USA}$ ) respectively. Slides were incubated overnight at $4^{\circ} \mathrm{C}$. A goat anti-rabbit lgG antibody conjugated with Alexa Fluor 488 (Invitrogen, Carlsbad, CA, USA) was used (1:100) for both primary antibodies. In the negative controls, the primary antibody was omitted and the specificity of the primary antibody was checked by using nonimmune rabbit lgG (Santa Cruz Biotechnology Inc.).

\section{Analysis of cellular localisation of LC3 and Beclin-1 by confocal microscopy}

Slides were observed under a fluorescence microscope connected to a Radiance plus Confocal Scanning system (Bio-Rad Laboratories, Hercules, CA, USA) for differential interference contrast (DIC) and fluorescence emission. Fluorescence signals were detected with $\mathrm{Ar} / \mathrm{He}$ laser diodes at a 488/568 emission wavelength.

Absence of localised fluorescence throughout the nucleus was confirmed by a z-scan of tissue (data not shown).

\section{Western blotting}

Proteins were extracted using RIPA buffer (Sigma-Aldrich, St. Louis, MO, USA) and quantified with the Bio-Rad Protein Assay (Bio-Rad Laboratories). Sixty micrograms of the total protein extract of each sample was separated by $15 \%$ (LC3) or 10\% (Beclin-1) sodium dodecyl sulphate polyacrylamide gel electrophoresis and electroblotted on polyvinylidene fluoride membrane (Mini Trans-Blot, Bio-Rad Laboratories). The transferred membranes were probed with rabbit polyclonal antibody against LC3 at 1:500 dilution (NB100-2220, Novus Biologicals) and with rabbit polyclonal antibody against Beclin-1 at a dilution ratio of 1:600 (Santa Cruz Biotechnology Inc.) at $4^{\circ} \mathrm{C}$ overnight. A goat anti-rabbit horseradish peroxidase conjugated secondary antibody (1:4,000, KPL, Baltimore, MD, USA) was added. Membranes were developed with ECL (Amersham Biosciences, Little Chalfont, UK). Polyclonal antibody against $\beta$-actin was used as a loading control (Sigma-Aldrich).

\section{Results}

We investigated the activation of autophagy in human first trimester and term placental villi by LC3 and Beclin-1 expression.

Slices from placental tissue were simultaneously analyzed with a LSCM for DIC and fluorescence emission. Confocal-DIC merged images showed a close correspondence between the fluorescent signals and the tissue structure (Figure 1G, 1L).

The negative control samples revealed a minimal green autofluorescent signal (Figure 1A, 1B, 1C, 1D).

To investigate whether autophagy was activated in human first trimester placental villi, we analyzed the expression of LC3 and Beclin-1 
Voluntary interruption of pregnancy (VIP)
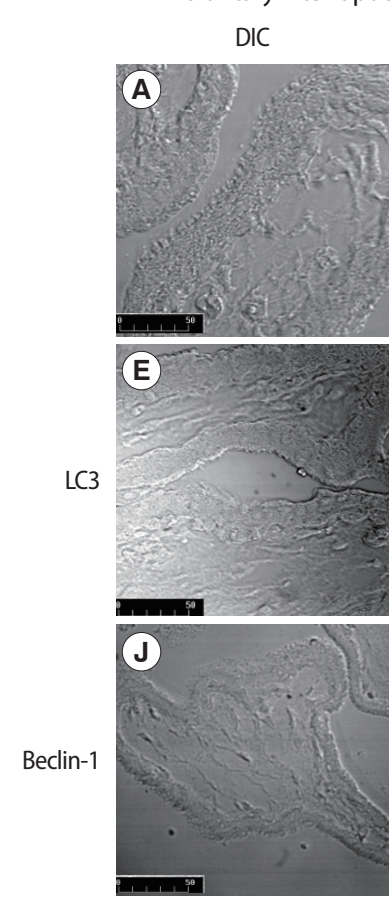

Fluorescence
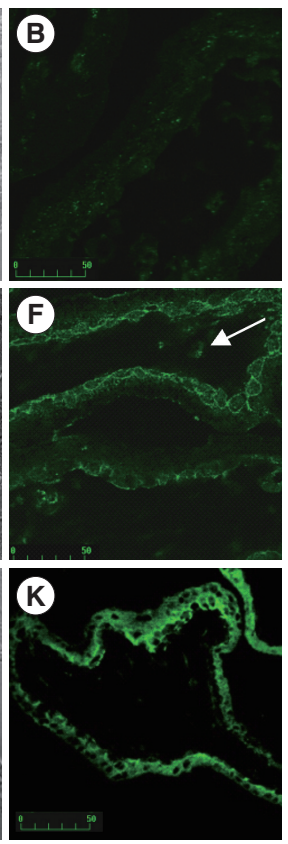

Term placenta (TP)

DIC
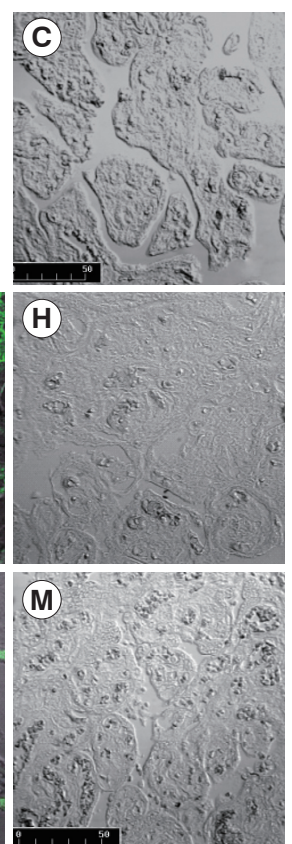

Fluorescence
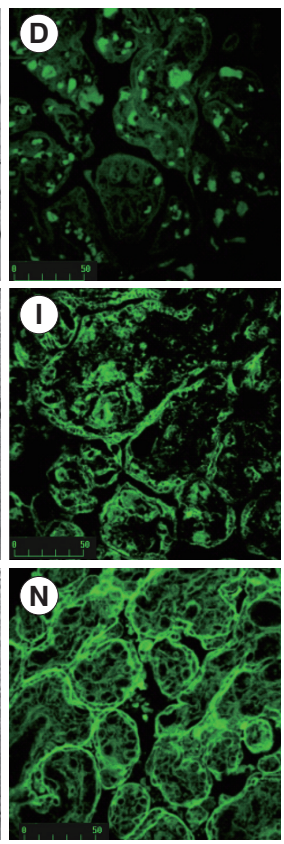
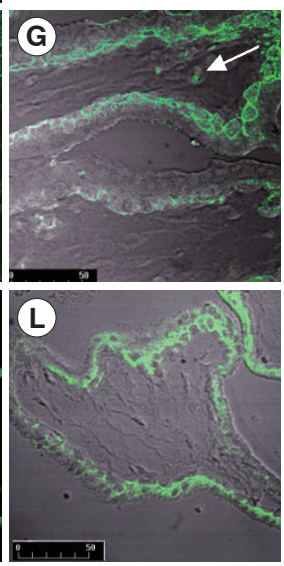

VIP

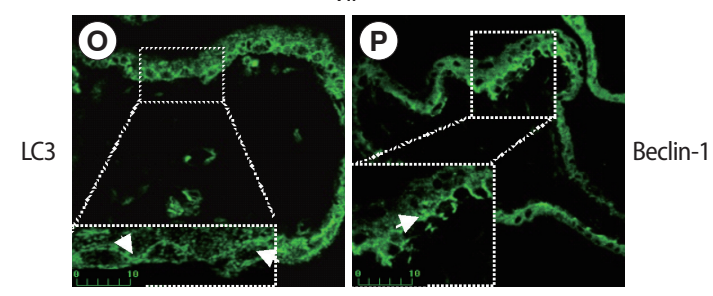

Q

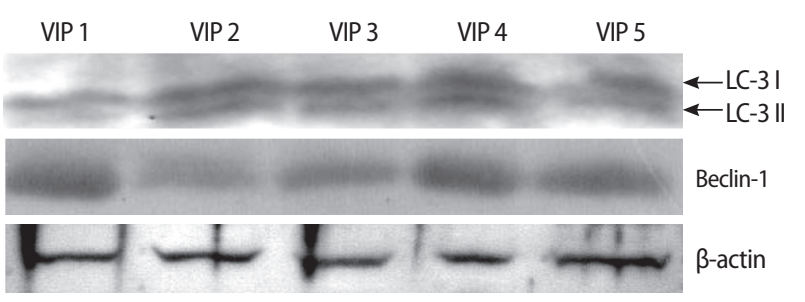

B

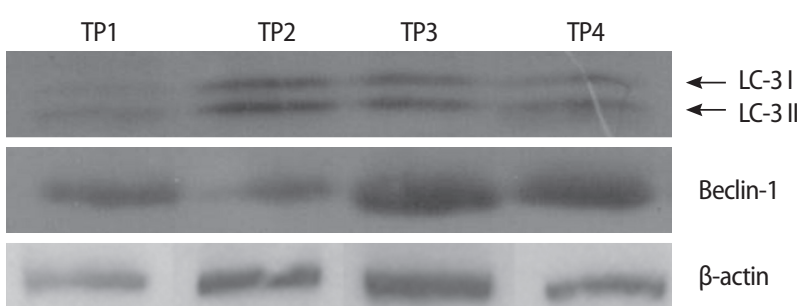

Figure 1. LC3 and Beclin-1 expression in human first trimester and term placental villi. (A-D) negative control samples; (B, D) green autofluorescent signals were minimal, (A, C) DIC images. x400; (F) LC3 punctate structures representing autophagic vacuoles are prevalently localized in the cytotrophoblast layer; LC3 positivity in stromal cells (arrows), (E) DIC image, (G) The correlated LSCM and DIC images are overlaid and merged. $\times 400$; scale bar is $50 \mathrm{~mm}$. (0) The inset shows a magnified view of the marked area, demonstrating the intense dot-like structures representing autophagic vacuoles (arrows). $\times 400 ; 3,6 \times$ electronic zoom; scale bar is $10 \mathrm{~mm}$. (N) Immunofluorescent staining for Beclin-1 reveals a diffuse localization in syncytiotrophoblast cell as well as punctate structures in cytotrophoblasts, (J) DIC image, (K) Beclin-1 immunoreactivity presented as faintly detectable fluorescence diffused in the cytoplasm of syncytiotrophoblast cells or as discrete fluorescent spots in the cytotrophoblasts, (L) The correlated confocal and DIC images are overlaid and merged. $\times 400$; scale bar is $50 \mathrm{~mm}$. (P) The inset shows a magnified view of the marked area, demonstrating the dot-like structures in cytotrophoblasts (arrows). $\times 400 ; 3,6 \times$ electronic zoom; scale bar is $10 \mathrm{~mm}$. (I) LC3 and, (N) Beclin-1 immunostaining from term placental villi shows LC3 and Beclin-1 localization in the trophoblast layer. (H, M) DIC images. (Q) Representative western blot for LC3 and Beclin-1 on human first trimester placental villi lysates. LC3-I soluble form of LC3 and LC3-II, the posttranslationally processed form of $\mathrm{LC} 3$, which localizes to autophagosomes and autophagolysosomes during autophagy, were distinguished. Five representative experiments are shown. (R) Immunoblots for LC3 and Beclin-1 on human term placental villi lysates show the conversion of LC3-I to LC3-II, confirming the autophagy activity. Four representative experiments are shown. LC3, light chain 3; DIC, differential interference contrast; LSCM, laser scanning confocal microscopy. 
by immunofluorescence and western blotting analysis.

Immunofluorescence and DIC merged images showed LC3-specific punctate dots prevalently localized in the cytotrophoblast layer compared to that of the syncytiotrophoblasts (Figure 1E, 1F, 1G). Under higher magnification, autophagic structures were clearly identifiable (Figure 10, arrows). Occasionally, we observed LC3 positivity in some stromal cells (Figure 1F, 1G, arrows).

Beclin-1 immunoreactivity presented as faintly detectable fluorescence diffused in the cytoplasm of syncytiotrophoblast cells or as discrete fluorescent spots in the cytotrophoblasts (Figure 1J, 1K, 1L). Under higher magnification, dot-like structures were clearly identifiable (Figure 1P, arrow).

Western blotting analysis showed the conversion of LC3-I into LC3II and Beclin-1 expression in all placental samples examined, confirming the autophagic activation (Figure 1Q).

LC3 and Beclin-1 immunostaining from term placental villi showed LC3 and Beclin-1 localization in the trophoblast layer (Figure 1H, 1l, $1 \mathrm{M}, 1 \mathrm{~N})$. The activation of LC3 and Beclin-1 expression were confirmed by western blotting analysis (Figure 1R). These findings were in line with previously published data in term placenta, so that they may act as an internal control for first trimester placental villi data.

\section{Discussion}

Accumulating evidence indicates an emerging role for autophagy in the pathophysiology of the human placenta [10]. Recent studies report that autophagy occurs in the human normal term placenta [14]. Placentas from preeclamptic and intrauterine-growth-restricted pregnancies have shown increased autophagy compared with normotensive pregnancies. In all these studies, autophagy was observed in syncytiotrophoblast cells [10-12].

These observations prompted us to investigate whether autophagy activation also occurred in early pregnancy. To this end, we analyzed LC3 and Beclin-1 expression by LSCM and western blotting in human first trimester placental tissue.

Upon induction of autophagy, LC3-II becomes associated with the autophagosomal membrane. This change in the subcellular distribution of LC3 can be followed by immunofluorescence microscopy and a characteristic pattern of LC3 puncta can be observed in autophagic cells stained with anti-LC3 antibodies.

In all of the samples analysed, immunofluorescence LC3-specific punctuate dots were clearly detected in cytotrophoblasts of human first trimester placental villi. This was not the case for the syncytiotrophoblast layer, where the LC3 staining, faintly and homogeneously distributed in the cells' cytoplasm, was indicative of absence of autophagic vacuoles. Occasionally, LC3 positivity also occurred in stromal cells; however, the significance of this finding is unclear.
Western blotting analysis confirmed the immunofluorescence data, showing LC3 activation in all samples analyzed.

To observe whether indeed the presence of autophagic vacuoles, visualized as LC3-positive puncta, reflected ongoing autophagy, we monitored Beclin-1 expression in the first trimester placental villi trophoblast layer.

The presence and the cytoplasmic distribution of Beclin-1, analyzed by immunofluorescence, showed a spot pattern only in the cytotrophoblasts of human first trimester placental villi, demonstrating signs of autophagy activation in these cells and not in the syncytiotrophoblast layer, where Beclin-1 fluorescence was present as a diffuse signal. The dot patterns of LC3 and Beclin-1 immunolocalisation were assumed to prove ongoing autophagy in the cytotrophoblasts of human first trimester placental villi.

Our results on autophagy activation in the trophoblast layer of human full term trimester placental villi were in line with previously published data.

In conclusion, our study showed for the first time that autophagy is already evident in the first trimester of pregnancy. In particular, LC3 and Beclin-1 immunofluorescence positivity mirrored an increased autophagy level in cytotrophoblast cells compared to the syncytiotrophoblast layer in first trimester placental villi.

In light of our findings, we hypothesize that, although autophagy may play a prosurvival role throughout human pregnancy, the need for autophagy may differ among cytotrophoblasts and syncytiotrophoblasts depending on the gestational age.

During early pregnancy, trophoblast stem cells, also referred to as cytotrophoblast cells, proliferate and differentiate by fusing to form the multinucleated syncytiotrophoblast layer. Therefore, in early pregnancy, autophagy might act as a cytoprotective mechanism in cytotrophoblasts, favouring stress adaptation during the challenging microenvironment of early placentation. Particularly, in the low-oxygen environment of the first trimester, autophagy may promote cell survival by antagonizing apoptosis and aiding in nutrient recycling, while promoting catabolic reactions of nutrients supplied by maternal plasma and secretions from maternal endometrial glands.

Later in pregnancy, syncytiotrophoblasts represent the interface between the maternal and fetal circulation, allowing gas and nutrient exchange when higher concentrations of oxygen are required to maintain the rapid rate of foetal growth. As a consequence, maintenance of the syncytium's integrity becomes highly important and autophagy might play an important role in homeostasis of the nondividing and highly differentiated syncytiotrophoblast cells.

The inherent limitation of this study may be the fact that it remains to be seen whether there are differences in week 8 as compared to week 12 , when oxygen concentration differs. In our future work, we will attempt to investigate the relationship of autophagy to stress 
and hypoxia in first trimester and term placental villi, studying the expression of proteins such as Beclin-1, damage-regulated autophagy modulator, p53, and HIF-1. We will also investigate autophagy in pathological placental tissues.

\section{Conflict of interest}

No potential conflict of interest relevant to this article was reported.

\section{Acknowledgments}

We thank Dr. Vincenzo Nardini and the staff at Anatomy Pathology Unit 2, Santa Chiara Hospital-University Health Care System Pisa.

\section{References}

1. Yang Z, Klionsky DJ. Eaten alive: a history of macroautophagy. Nat Cell Biol 2010;12:814-22.

2. Cecconi F, Levine B. The role of autophagy in mammalian development: cell makeover rather than cell death. Dev Cell 2008;15: 344-57.

3. Kabeya Y, Mizushima N, Ueno T, Yamamoto A, Kirisako T, Noda T, et al. LC3, a mammalian homologue of yeast Apg8p, is localized in autophagosome membranes after processing. EMBO J 2000; 19:5720-8.

4. Liang $X \mathrm{XH}$, Kleeman LK, Jiang HH, Gordon G, Goldman JE, Berry G, et al. Protection against fatal Sindbis virus encephalitis by beclin, a novel Bcl-2-interacting protein. JVirol 1998;72:8586-96.

5. Yue Z, Jin S, Yang C, Levine AJ, Heintz N. Beclin 1, an autophagy gene essential for early embryonic development, is a haploin- sufficient tumor suppressor. Proc Natl Acad Sci U S A 2003;100: 15077-82.

6. Liang XH, Jackson S, Seaman M, Brown K, Kempkes B, Hibshoosh $\mathrm{H}$, et al. Induction of autophagy and inhibition of tumorigenesis by beclin 1. Nature 1999;402:672-6.

7. Liu GH, Zhong Q, Ye YL, Wang HB, Hu L, Qin ZK, et al. Expression of beclin 1 in bladder cancer and its clinical significance. Int J Biol Markers 2012 Nov 5 [Epub]. http://dx.doi.org/10.5301/JBM.2012. 9769.

8. Wirawan E, Lippens S, Vanden Berghe T, Romagnoli A, Fimia GM, Piacentini $M$, et al. Beclin1: a role in membrane dynamics and beyond. Autophagy 2012;8:6-17.

9. Huppertz B. The anatomy of the normal placenta. J Clin Pathol 2008;61:1296-302.

10. Signorelli P, Avagliano L, Virgili E, Gagliostro V, Doi P, Braidotti P, et al. Autophagy in term normal human placentas. Placenta 2011; 32:482-5.

11. Oh SY, Choi SJ, Kim KH, Cho EY, Kim JH, Roh CR. Autophagy-related proteins, LC3 and Beclin-1, in placentas from pregnancies complicated by preeclampsia. Reprod Sci 2008;15:912-20.

12. Curtis $\mathrm{S}$, Jones $\mathrm{CJ}$, Garrod $\mathrm{A}$, Hulme $\mathrm{CH}$, Heazell $\mathrm{AE}$. Identification of autophagic vacuoles and regulators of autophagy in villous trophoblast from normal term pregnancies and in fetal growth restriction. J Matern Fetal Neonatal Med 2013;26:339-46.

13. Hung TH, Chen SF, Lo LM, Li MJ, Yeh YL, Hsieh TT. Increased autophagy in placentas of intrauterine growth-restricted pregnancies. PLoS One 2012;7:e40957.

14. Bildirici I, Longtine MS, Chen B, Nelson DM. Survival by self-destruction: a role for autophagy in the placenta? Placenta 2012; 33:591-8. 